\title{
Gendern im Deutschen
}

\author{
Rudolf WINDISCH \\ Prof. Dr. Dres hc.; Universität Rostock; \\ E-Mail: rudolf.windisch@yahoo.de
}

\begin{abstract}
The terms gendern (< engl. gender) or gendering in German stand for the attempt to establish equality between men and women in everyday life or in political controversy, in academic life by means of linguistic methods. The representatives (plural feminine) of the feminist language criticism/ Feministische Linguistik call for the dissolution of the "generic masculine" for ex. jeder Lehrer (masculine/feminine) means a gender-mixed group whose gender/sex is neither relevant, nor known. It is criticized that the female teacher is not explicitly mentioned. The question here is whether the desired gender-appropriate treatment of all women germ. das Gendern - the genderconscious use of language - is guaranteed by diacriticalorthographic procedures such as internal I: LehrerInnen (fem. pl.), slash (/): Leser/innen (masc.sg./pl.;/f.pl.), asterisk shape: Lehrer*innen or gender-gap: ein_e Beamt_er_in (an official, m./f. sg.) or by replacement of pair shapes like die Arbeitnehmenden (employees; instead of the "classic form' Arbeitnehmer/Arbeitnehmerinnen) or the participial construction die Fahrzeugführenden/ the vehicle-carrying/ drivers.
\end{abstract}

Key Words German verb forms gendern/gendering, feminist language criticism, equality between men and woman, dissolution of the generic masculine, linguistic procedures for gender-equal treatment

Was verstehen Deutsch-Sprecherinnen und Sprecher darunter? Mit der denominalen Ableitung Gendern von engl. gender 
„Geschlecht“" oder gendering, ,Vergeschlechtlichung“, wird die gesellschaftlich-soziale Gleichstellung von Mann und Frau im Alltagsleben, im zwischenmenschlichen Kontakt, am Arbeitsplatz, in der politischen Auseinandersetzung oder im wissenschaftlichen Sprachgebrauch angestrebt, insgesamt also ein weiter Bereich, in dem eine Feministische Sprachkritik am heutigen Alltags-Schriftdeutsch anknüpft. Der Zugang zu diesem steinigen Feld steht dem interessierten Leser-Sprecher an beliebiger Stelle offen, wie exemplarisch etwa aus den Darlegungen bei Opitz-Belakhal zum Thema Geschlechtergeschichte und der dort angeführten Literatur zum Gendering hervorgeht. Laut Opitz-Belakhal wird in den Geschichts- und Sozialwissenschaften mit Gendering ein Thema unter einer geschlechterspezifischen Fragestellung und Perspektive untersucht (...): „Dabei wird davon ausgegangen, dass Geschlecht in nahezu allen Lebensbereichen eine Rolle spielt und Herrschaftsverhältnisse geschlechtlich markiert sind. Geschlecht prägt Denken, Vorstellungen, die soziale und politische Welt und diese konstituieren das soziale Geschlecht (Gender).“

An welche längst verschwundenen Herrscher (gab es keine HerrscherInnen?) oder Herrschaften sollte man sich heute noch erinnern? Und ist der Satz evident: „Geschlecht prägt Denken“ - vielleicht eher so, dass es die sozial- und kulturgeschichtlichen Erfahrungen sind, die beide Gender prägen? Historisch belegt sind die unzähligen Berichte über den von jenen Herrschern alltäglich verübten Machtmissbrauch, der immer noch, in totalitären Staatsformen, auch unter dem Druck religiöser Riten, das Lebens der sozial ,niederen“ Schichten Jahrhunderte lang, bis heute, beeinträchtigte. Noch zu Beginn des 20. Jahrhunderts zeigte sich der überkommene virile Machtanspruch bei der Frage nach der Zulassung von Frauen zum Studium: in Preußen wurden ihnen 1896 zunächst recht großzügig Zutritt als Gasthörer gewährt, erst ab 1908 als Studierende. Bekannt ist der gegen die männliche Usurpation gerichtete Widerstand 
emanzipierter Frauen, wie etwa der von Agnes von ZahnHarnack (Tochter des Kirchenhistorikers Adolf von Harnack, 1851-1930), die sich in Berlin als eine der ersten Frauen an der Universität einschreiben ließ und als eine der wichtigsten Persönlichkeiten der Frauenbewegung bekannt wurde.

Wie die von C. Opitz-Belakhal (vgl. Anm.1, Geschlechtergeschichte 2010: 45) zitierte Arbeit der amerikanischen Wissenschaftshistorikerin Londa Schiebinger zeigt, setzte diese Bewegung, auch außerhalb Deutschlands, innerhalb der Naturwissenschaften im Kontext der ,vorherrschenden Geschlechterbilder und -dichotomien" bereits um 1800 ein - aber war diese ,maskuline“ Vorherrschaft dort tatsächlich bereits verpönt, während sie sich in Berlin oder in München noch auswirkte? Die im Rahmen einer sozial-politischen Rückschau entworfene Geschlechtergeschichte widmet sich längst den Fragen einer geschlechtsspezifischen Arbeitsteilung, feministischer Wissenschaftstheorien, frauenspezifischer Aktivitäten im Universitätsbetrieb usw. - ein für Außenstehende weitgefasstes und nur schwer überschaubares Feld der Gender-Forschung. Bleibt die Frage, ob es im Verlaufe der langen, sozial- und machtpolitisch geprägten Männer-Herrschaft nicht doch auch frühe Lichtblicke weiblicher Durchsetzungskraft gab? Man denkt - exemplarisch - etwa an die letzte Medici, an Anna Maria Luisa (1667-1743) als Sammlerin wertvoller Kunstgegenstände in ihren Residenzen, zuletzt in Florenz, wo sich ,[...] visuelle und materielle Hofkultur zu einem Mikrokosmos weiblicher Herrschaftsinteressen innerhalb der vordergründig männlich dominierten toskanischen Hofkultur [verdichten].“ $\mathrm{Zu}$ erinnern wäre auch an Maria Theresia, Erzherzogin, Königin, Großfürstin (17171780), die in der Nachfolge ihres Gatten Franz I. Stephan (ab 1745 römisch-deutscher Kaiser, $† 1765)$ die Staatsgeschäfte der Habsburger-Monarchie besorgte.

Wie aber lassen sich mit Hilfe einer grammatikalisch angelegten Gleichstellung von mask./fem. die Verstöße gegen die 
Achtung der längst angestrebten Gleichstellung von „Frau“/ „Mann“ in der Arbeitswelt, in der Forschung, im Alltag, ahnden? Setzt der Respekt vor dem sozial bestimmten GenderUnterschied nicht zuallererst eine gegenseitige persönliche Achtung voraus, die sprachliche Sorgfalt im wechselseitigen Austausch, in einem neuen ,Sprech-Stil'? Die engagierten Verfechter der Ausarbeitung jener proklamierten gendergerechten Sprache vertreten offensichtlich die These, dass Sprache die Wahrnehmung lenke, also die sozial ,korrekte' er/Mann - sie/ Frau-Unterscheidung. Aber widerspricht diese der Sprache zugeschriebene Leistung nicht einer überlieferten, von der Sprachphilosophie geprägten Vorstellung, wonach sie doch Ausdruck des Denkens selbst ist, durch das sie auch bestimmt wird? Nur beruht das grammatikalische Mitmeinen von Mann ,mit' Frau weniger auf philosophischer Einsicht, denn auf der für das Indogermanische grundlegenden Sexus (natürliches Geschlecht) / Genus (grammatikalisches) - Klassifikation von Mensch und Tier. Wieweit diese animistische, d.h. die auf männlich - weiblich ausgerichtete Sex-Klassifikation der Lebewesen dann auf die gesamte Nominalbildung der Sprache ausgeweitet wurde, bleibt im Dunkel der Geschichte. Laut Wilhelm Wundt (1832-1920) wurde ,dadurch ihre ursprüngliche Bedeutung offenbar sehr frühe schon gänzlich verwischt (...).“ Für Hermann Paul (1846-1921) gilt in dieser Hinsicht:

Die Basis für die Entstehung des grammatischen Geschlechts bildet der natürliche Geschlechtsunterschied der menschlichen und tierischen Wesen. Wenn außerdem noch anderen Wesen, auch Eigenschafts- und Tätigkeitsbezeichnungen ein männliches oder weibliches Geschlecht beigelegt wird, so ist das eine Wirkung der Phantasie, welche diese Wesen nach Analogie der menschlichen Persönlichkeit[...] (Paul 1920)

Im Verlaufe der Sprachentwicklung verliert sich die ursprüngliche Übereinstimmung von Geschlecht/Genus mit biologischem Sexus und wurde „noch anderen Wesen beigelegt“ (Ebd.): der 
Mann/ Löwe/ Baum, die Magd/ Giraffe/ Sonne; das Neutrum

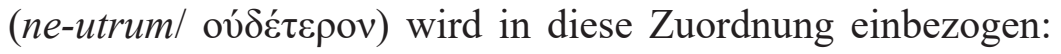
das Pferd/ Wasser/ Mädchen usw. Sieht man von H. Paul ab, so belegt ein Blick auf andere Sprachen dieser Welt, dass die indogermanische Nominalklassifikation nur eine unter vielen möglichen Wort-Ordnungen darstellt. Im historischen Abstand zu jener frühen Klassifikation gilt es längst - wie die zahlreichen Abhandlungen über das Gendering/Gendern belegen -, das heutige Deutsch im Sinne seines geschlechterbewussten, gendergerechten Einsatzes zu verteidigen bzw. auszubauen. Wie schauen diese genderinkludierenden Regeln und ihre Formen bei der Beachtung einer geschlechtergerechten Sprache im Alltag, in der Sprache der öffentlichen Medien, in Kunst und Wissenschaft heute aus? Zur Umsetzung dieses Anspruchs gilt der Verzicht auf das sog. generische Maskulinum (kurz: gener. Mask.), ein sprachlich-linguistisches Verfahren, wenn männliche Bezugspersonen, z.B. Lehrer (mask. Nomina und Pronomina), zur Benennung gemischtgeschlechtlichen Gruppen angeführt werden. Der Satz, ,jeder Lehrer freut sich, wenn er gelobt wird“, schließt alle männl. und weibl. Lehr-Personen ein, deren Geschlecht hier nicht von Bedeutung oder unbekannt ist. Wie aber gendert man richtig, um der feminist. Kritik am gener. Mask. wegen eines „Nicht-Mitgedachtwerden“" weibl. Personen zu widersprechen? Böte hier die „Vormünderin“ aus einem Berliner Jugendamt ein Vorbild? Oder wäre die „Vorständin" an der Wiener Universität nicht doch eleganter? Was die Sprache ihren SprecherInnen nicht alles erlaubt!

Die feministische Linguistik stellt die Bezeichnung gemischtgeschlechtlicher (Berufs-) Gruppen in den Mittelpunkt ihrer Untersuchungen, bei denen das Geschlecht (Sexus) der Personen nicht genannt bzw. vordergründig nicht von Interesse ist: ,jeder Lehrer will guten Unterricht machen“, bezieht sich im allgemeinen Sprachgebrauch immer noch sowohl auf weibliche wie auch männliche Personen; dabei wäre das gener. 
Mask. der bisher üblichen geschlechtsneutralen Formen wie Schulkinder durch Paarformen mit Nennung beider Geschlechter wie Schüler/Schülerinnen zu ersetzen; allerdings decken sich diese ,neutralen' Formen semantisch nicht eindeutig mit dem zugrundeliegenden gener. Mask.: „Wählende“ müssen keine (politischen) „Wähler“ sein, „Studierende“ keine (Univ.-) „Studenten“. Schwierigkeiten bei den Alternativen zum gener. Mask. ergeben sich bei jenen Komposita, bei denen eines die Basis bzw. den Modifikator bildet, z.B. Bürgermeister $>$ *Bürgerinnenmeisterinnen; wie verhält es sich bei der Bildung der Adjektive aus fem. Basis, z.B. Kauffrau $>*$ kauffraulich, Richterin $>$ *richterinlich wie mask. richterlich, fachmännisch? Nun ist in sozial markierten Alltagswörtern wie Lehrerin die formale-begriffliche Unabhängigkeit vom gener. Mask. längst erreicht, sicherlich auch bei der Bundeskanzlerin. So lässt sich heute verfolgen, wie es bei Berufsbezeichnungen (Schuster) und bei den (deverbalen und/oder denominalen) Nomina agentis (kaufen> Käufer/ Stadt> Städter) seine Vorrangstellung eingebüßt hat, ganz im Sinne der von der feministischen Linguistik angestrebten sprachlichen Gleichstellung von m./f.; es teilt nun seinen Einsatz mit den genannten Alternativen wie Doppelnennungen (Mitarbeiter und Mitarbeiterinnen), den Umschreibungen z.B. alle, die für die die Stadtverwaltung arbeiten, oder mit typographischer Markierung wie einem Binnen-I usw. Damit scheint die Dominanz des Mask. geschwächt, wenn nicht bereits ausgehebelt. Lässt sich nun bei der Konstruktion gendergerechter Formen das Fem. ohne Rücksicht auf sprachliche Umgangsformen und sozialpolitische Bedenken - automatisch einrichten? Ableitungen wie *FlüchtlingInnen (< Flüchtling/e, m./f. pl.), AsylantInnen $<$ Asylant/en oder VerbrecherIn, TerroristIn wird man dem ,Weiblichen' nur ungern nachrufen, ,man' ist ja höflich! Dagegen kennt Flüchtling keine fem. Form, eine Nominalisierung mittels des Part. Präs. flüchtend = Flüchtende (sg. m./f.) würde 
aber nicht die Gemeinte, d.h. *eine Flüchtling bezeichnen; ähnlich verhält es sich mit „Leser“, der/die nicht mit „Lesende“ (m./f.) gleichzusetzen ist, wenn ersterer gerade jetzt liest, „die anderen haben vielleicht vor einem halben Jahr zum letzten Mal ein Buch gelesen. Im Übrigen wird, sobald sich zur Singularform der bestimmte oder unbestimmte Artikel gesellt, das Geschlecht schon wieder unbarmherzig enthüllt."

Wie steht es um ein/das generische(s) Femininum als (lexikalischem) Äquivalent zur Dominanz des gener. Mask. im Deutschen? Wir benutzen Feminina wie „Wache, Berühmtheit, Koryphäe, Waise" u.a., ohne das Geschlecht der betreffenden Wörter zu nennen, das in ihrem Kontext offensichtlich nicht von Bedeutung ist. Die femin. Tierbezeichnungen wie Amsel, Eidechse, Fliege, Forelle u.a. dürften das Gendern überfordern, da ein ,männliches' Pendant nicht zu bestimmen ist; ähnlich verhält es sich bei der genuin femin. Amme, zu der (bisher) kein *der Amme-r generiert wurde, während sich zur Elfe ein Elf gesellte. Die Ergebnisse eines „Geschickt Gendern“ sollten laut Vertretern dieser Disziplin in einem - zum DUDEN in Konkurrenz stehenden? - Genderwörterbuch auch festgehalten werden. Dort finden sich, alphabetisch geordnet, 956 Zeilen (= Beispiele) wie Abteilungsleiter (sg.) = Abteilungsleitung, (pl.) Abteilungsleitungen; Abteilungsleitende (m./w.pl.?); Akademiker $(\mathrm{pl}$.) = Studierte (könnte regional auch ironisch, abwertend gebraucht werden); ist Absender (sg./pl.) mit abgesandt durch/von $[\mathrm{XY}]$, geschickt' gegendert? Reicht auf dem Briefumschlag nicht, wie bisher, der Name? Nicht, dass versehentlich Abgesandte+Name vermerkt wird! Oder: Absolvent (sg./pl.) =Abschlussinhabende[dasPC-Rechtschreibeprogramm streikt] Personen/ mit Abschluss, usw. - Cui bono?

Ein eigenes Kapitel bei der Schreibung einer geschlechtergerechten Sprache bilden die Markierungen wie z.B. Bürger*innen, Jüd*innen (vgl. SZ, 01.04.2020, S. 9; Jüd-?) mit dem sog. Gendersternchen - laut einer Kritik des Vereins 
Deutsche Sprache (VDS) ,im Prinzip auch nix anderes als fünf Deppenapostrophe in kreisförmiger Anordnung" oder die Setzung des Gendergap (Lücke), z.B. ein_e Beamt_er_in (sic!). Beide Formen kritisiert der DUDEN wie auch der VDS. Zahlreich sind auch die Formen mit Binnenmajuskel wie VertreterInnen, oder mit Schrägstrich ein/-e Beamter/-in usw. Wie können diese gendergerechten Graphien in den Nachrichten oder im TV, auch „,in leichtem Deutsch“, bei der ,Übersetzung“ für Gehörlose durch die bewundernswerte, für den normalen Seher-Hörer nicht verstehbare, lautlose Gestik-Mimik der Übersetzer/innen wiedergegeben werden? In den Nachrichten hört man heute KollegInnen mit einem glottal-stop. Bei der Umsetzung der neuen Sprachformen deutet der Journalist Schloemann auf die Stadtverwaltung von Hannover, die am 18. Januar 2018 ihre „Stadtverwaltenden“" zu einer geschlechtergerechten Sprache im Schriftverkehr mit den Bürgern anwies, das gener. Mask. aus der Kommunikation herauszunehmen und alle Bürger*innen in geschlechtergerechter mask. oder fem. Form anzusprechen; auch seien Generica wie ,jeder" (ein jeder/ eine jede) durch „alle“ (Männer-Frauen?) zu ersetzen. Schloemanns Fazit: „Hannover wird geschlechtergerechter.“ Auf die Hannoveranische amtssprachliche Regelung geht auch Helmut Glück ein und erinnert daran, dass „Staatsbehörden [...] jedoch nicht schreiben [dürfen], wie sie wollen" und sich an die im Verwaltungsverfahrensgesetz ( $V w V f G$, vgl. $§ 23$ Amtssprache) festgelegte amtliche Rechtschreibung zu halten hätten: „Die Stadt Hannover setzt sich darüber hinweg.“ (vgl. Anm. 14) Könnte man die Pflege einer geschlechtergerechten bzw. hierarchiebewussten Sprache - auch ohne kritisches Vorurteil - nicht doch auch unter Rückgriff auf traditionelle Sprach- und Schreibformen abwickeln? Wenn die, Wählerinnen und Wähler" bei den Nationalratswahlen in Österreich am 20. September 2019 zur Abgabe ihrer Stimme im TV/Rundfunk aufgerufen wurden, so hat man sich als $\mathrm{Zu}-\mathrm{Hörer}$ längst an die 
simple, zeitraubende Gleichstellung von f./m. gewöhnt: hier schlägt das o.g. Genderwörterbuch nun den Verzicht auf ,eher umständliche Paarformen" wie Mitarbeiterinnen und Mitarbeiter vor und bietet partizipiale Ableitungen an, wie die Mitarbeitenden, die Arbeitnehmenden (so heute längst in Betrieben), die Studierenden (einer Universität), die Fahrzeugführenden (so in der dt. StVO) oder die Radfahrenden - alles ,Neusprech“? Wieso darf es kein-e Radler*in sein, welche/r gerade auf den Kurfürstendamm einbiegt? Bei der durch das Gendering angestrebten Verdrängung des gener. Mask. wäre zu berücksichtigen, dass die historisch überlieferten mask. Berufsbezeichnungen, wie Förster, Pfarrer, Müller u.a. nicht primär sexusmarkiert sind; es ließe sich ja - wenn auch berufsmäßig noch nicht üblich - eine Försterin ableiten, während die Pfarrerin-nen in der evangelischen Amtskirche längst schon ihren Dienst angetreten hat/haben. Dagegen sind die Nomina agentis auf -in/-esse/isse/-euse, z.B. Visagistin/ Hostesse/ Diakonisse oder Diseuse exklusiv feminin. Hat das Maskulinum hier keine oder nur wenige Chancen auf eine Umsetzung - vgl. Souffleuse / Souffleur - etwa der *Diseur? Laut Helmut Glück ist das gener. Mask. „ein Mechanismus, der auch für den Satzbau wichtig ist. Er regelt, dass in einigen Fällen das Maskulinum als regierendes Genus fungiert [...] etwa bei Fragepronomen (Wer hat seinen Lippenstift im Bad liegenlassen?)... . Auch Wörter wie deswegen und indessen beruhen auf maskulinen Formen. Das Maskulinum ist in solchen Fällen sexusneutral. Es ist hier (und anderswo) das unmarkierte Genus, das alle Sexus bezeichnet.“

Dagegen ist Senta Trömel-Plötz davon überzeugt, dass „,das vermeintlich geschlechtsneutrale generische Maskulinum ... eben nicht geschlechtsneutral wirke ..., sondern Frauen gedanklich auslösche.“ Ist also im weiten Berufsfeld „Arzt" eine weibliche Kollegin nicht mitgemeint? Welche Begründung für die Auflösung des gener. Mask. zugunsten einer geschlechtergerechten Sprache will man noch anführen? Unter Missachtung 
des DUDEN erfreut sich die - *innen Schreibung auch in staatlichen Kunstprogrammen größter Beliebtheit, wenn Archäo-

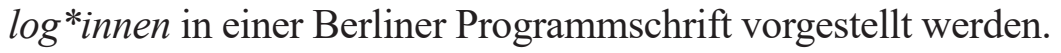
In den traditionell geschlechtsmarkierten Berufsbezeichnungen, wie z.B. Bäcker/ Bäckerin soll das fem. -in angeblich nicht einfach vom Maskulinum ableitbar sein: in einer von mehreren LeserInnen-Zuschriften in der SZ (6./7. April 2019, S. 14) auf der Forumsseite „Ein Sternchen, das spaltet“ mit Bezug auf vorausgehende Beiträge wie „Von Bäckern, Lehrerinnen und Flüchtlingen", glaubt eine Leserbrief-Schreiberin an den beruflichen Vorrang der Bäckerin gegenüber einem Bäcker: „Die Bäckermeister waren bis zum 19. Jahrhundert Frauen, eben Bäckermeisterinnen. Mehr und mehr drängten Männer in diesen Beruf, verdrängten (...) die Frauen und tilgten sie mit der Zeit aus der Sprache. (...) Und das ist nur ein Beispiel, wie die Sprache Wirklichkeit abbildet, aber auch formt.“

$\mathrm{Ob}$ sich aber aus der sozialgeschichtlichen Entwicklung dieses Metiers (und vergleichbar anderer) linguistisch verwertbare Aussagen über die Gestaltungskraft der Sprache hinsichtlich einer Gleichberechtigung bzw. Missachtung von mask./ fem. formulieren lassen? Für das Ungarische (Gender Studies sind in Ungarn als Studiengang nicht zugelassen) stellt sich die Frage nach einem einzuklagenden Bäckerin-Primat erst gar nicht: beide, der/die Bäcker/Bäckerin, lauten a pék. Zweifellos war es laut Markus-Evangelium (16,1-8) ein frühes feministisches Primat, dass gerade Frauen den Jüngern Jesu an Ostern die Kunde vom leeren Grab des Herrn zutrugen.

Wieweit gilt es nun laut Gender-Gleichheit in der überlieferten Arbeitswelt, in der Männer schon immer handwerkliche Schwerstarbeit lieferten, wie etwa der Schmied, auch eine Schmied*in (professionell noch nicht aktuell?) zu respektieren; und arbeitet selbige auch *fach fraulich? Ist sie nun eine *Ränke_Schmiedin oder die wohlgeachtete „Frau Schmied“? Dabei reicht die Geschichte der weiblichen Arbeitswelten weit 
in das Mittelalter zurück: „Gearbeitet haben Frauen natürlich schon immer. Sichtbar waren sie dabei lange nicht. Und unbezahlt noch dabei. Eine Chronologie von der Antike bis heute." Zahlreiche Belege für Respektlosigkeit gegenüber Frauen, die Missachtung ihrer persönlichen Rechte, finden sich alltäglich, in der Arbeitswelt, im kulturellen Schaffen, im Bereich der künstlerischen Gestaltung der Musik-, Theater- und Filmwelt, wobei der verlangte Respekt sicherlich nicht erst durch die Anmahnung gendergerechter Sprachformen zu erwirken wäre, sondern im freundlich-persönlichen Umgang.

Eine kurze Auflistung der wichtigsten Pro- und ContraArgumente zum Gendern belegt eine quasi gleichwertige Relevanz bei den Methoden einer sozialen und sprachlichen Gleichbehandlung beider Geschlechter in allen gesellschaftlich relevanten Benennungen, Fachbezeichnungen oder bei akad. Titeln: „Frau Professor“" oder ,korrekt" „Frau -/in“, oder bei politischen Ansprachen etwa Frau Minister-in oder ganz allgemein: „liebe Bürgerinnen und Bürger"? Beim Streben nach einem solchen Ausgleich handelt es sich laut konservativer, rechtsgerichteter politischer Kreise um ein „Orwell-Projekt“, um „Genderwahn“, wie Alice Weidel, Mitglied im Deutschen Bundestag, provozierend meint. Allerdings sollte eine Kritik an der Gender-Linguistik auf solche Übertreibungen verzichten, solange wir alle (m./f.), ohne Verwarnung durch ,SprachpolizistInnen', unsere Muttersprache und unsere in der schulischen Ausbildung verbesserten Sprachkenntnisse, kontrolliert durch das gesellschaftlich-soziale Umfeld, einsetzen.

Wer-neben den bekannten feministischen Verfechter*innen eines gendergerechten Ausbaus der Sprache - könnte/sollte hier für eine verbindliche Normierung des Deutschen sorgen? Wir haben in Deutschland keine der Académie Française entsprechende zentrale Einrichtung. Sprachliche Normierungen werden von den bekannten Institutionen wie der DUDEN-Redaktion, der Darmstädter Akademie für Sprache und Dichtung, von dem 
Mannheimer Institut für Deutsche Sprache und vom Rat für deutsche Rechtschreibung übernommen. Alle Deutsch-Sprecher/Sprechende und Schreibende sollten deren Empfehlungen beachten. Offensichtlich bleibt hier noch einiges zu tun, liest man den exemplarischen Beitrag von Frau Prof. Maria Wersig (Präsidentin des Deutschen Juristinnenbunds): „Mehr Struktur! Die Gleichstellung in Deutschland geht schleppend voran. Auch weil dafür seit 25 Jahren professionelle Strukturen fehlen." Frau Wersig verweist u.a. auf die vor 25 Jahren vom Gesetzgeber in den Gleichberechtigungsgrundsatz Art 3, (2) des Grundgesetzes eingefügte Ergänzung: „Der Staat fördert die tatsächliche Durchsetzung der Gleichberechtigung von Frauen und Männern und wirkt auf die Beseitigung bestehender Nachteile hin." Von der Umsetzung dieses Verfassungsauftrags ist der Staat, laut Frau Wersig, noch weit entfernt.

Harald Martenstein zählt bei der Lektüre der Einführung in die Gender Studies von Franziska Schößler (2008) in seinem Beitrag "Geschlechterforschung: Schlecht, schlechter, Geschlecht" die Wörter Gender, Gender Mainstreaming und Gender Studies auf, unter denen sich die meisten Leute nicht viel vorstellen könnten. Dem darf man zustimmen, aber nicht der Vorstellung der Vertreter jener Gender Studies, die Martenstein ironisch-sarkastisch aufgreift (ebd.): „Genderforscher glauben, dass „Männer“ und „Frauen“ nicht eine Idee der Natur sind, sondern eine Art Konvention, ungefähr wie die Mode oder der Herrentag" - eine ,Konvention"? Tragen die ,natürlichen' Merkmale nun nicht mehr zum Unterschied Mann/Frau bei? Die Frage scheint erlaubt, versucht man Schößlers, von Martenstein zitierte (ebd.) Sicht zu verstehen: „Es sind vor allem kulturelle Akte, die einen Mann zum Mann machen.“ und was macht die Frau zur Frau? - Beginnen wir unsere nächste kollegiale Interaktion mit einem freundlichen „Grüß Gott Herr Professorin"! 


\section{Literaturverzeichnis}

Glück, Helmut: Die Ersatzreligion der sprachlichen Anbiederung. In: Sprachnachrichten. Verein Deutsche Sprache e.V. Heft 81 (I/2019).

Schößler, Franziska: Einführung in die Gender Studies. Berlin: Akademie-Verlag 2008.

Opitz-Belakhal, Claudia: Geschlechtergeschichte. (=Historische Einführungen). Frankfurt/M.: Campus Verlag 2010; ${ }^{2} 2018$.

Paul, Hermann: Prinzipien [Principien,1880] der Sprachgeschichte. Halle a. S.: Niemeyer 1920.

Royen, Gerlach: Die nominalen Klassifikationssysteme in den Sprachen der Erde. Historisch-kritische Studie, mit besonderer Berücksichtigung des Indogermanischen.

(Linguistische Anthropos-Bibliothek, Band IV), Mödling bei Wien 1930.

Schiebinger, Londa: Has Feminism Changed Science? Cambridge Mass.: Harvard University Press 1999.

Stollberg-Rilinger, Barbara: Maria Theresia: Die Kaiserin in ihrer Zeit. Eine Biographie. München: C. H. Beck 2017.

Windisch, Laura: Kunst. Macht. Image. Anna Maria Luisa de Medici (1667-1743) im Spiegel ihrer Bildnisse und Herrschaftsräume. Wien Köln Weimar: Böhlau Verlag 2019.

Wundt, Wilhelm: Völkerpsychologie (Sprache, Mythus und Sitte), 2. Band: Die Sprache, 2. Teil. Leipzig: Wilhelm Engelmann ${ }^{3} 1912$.

\section{Internetquellen}

Gendern. In: Wikipedia, die freie Enzyklopädie, unter: https:// de.wikipedia.org/wiki/Gendering (17.09.2019)

Generisches Maskulinum. In: Wikipedia, die freie Enzyklopädie, unter: https://de.wikipedia.org/wiki/Generisches Maskulinum (13.8.2019)

Frydrych, Gabriele: Windsbraut und Hackepeter - wie gendern wir richtig? In: Auswege. Perspektiven für den 
Erziehungsalltag vom 16. November 2019, unter: https:// www.magazin-auswege.de/2019/11/wie-gendern-wir-richtig/ (15.12.2019).

Gewerkschaft Erziehung und Wissenschaft Berlin. Unter: "http://www.gew-berlin.de/22112_ 23955.php"ew-berlin. de/22112_23955.php? (15.12.2019)

Steinfeld, Thomas: Geschlechtergerechtes Deutsch? Grammatikalisch unmöglich! In: Süddeutsche Zeitung online vom 15.03.2019, unter: https://www.sueddeutsche.de/kultur/ gender-geschlechtergerechte-sprache-verein-deutschesprache-1.4365716 (24.06.2019)

Academic: Generisches Femininum. Unter: https:/deacademic.com/dic.nsf/dewiki/504418(15.07.2019)

Das Genderwörterbuch/ geschicktgendern.de. unter: https:// geschicktgendern.de (15.07.2019)

Gendern.de - das größte Wörterbuch für eine gendergerechte

Sprache. unter: www.gendern.de (24.06.2019)

Das Deutsche als Männersprache. Friederike Sittler im Gespräch mit Luise F. Pusch. In: kulturradio vom 7.04.2019 und Pusch, Luise F.: Generisches Feminin statt Gendersternchen. In: taz vom 6.02.2019, beide unter: https:// geschicktgendern.de/tag/luise-f-pusch/ (24.06.2019)

Verein Deutsche Sprache. e.V.: Sprachnachrichten Nr. 81, 1/2019, unter: https://vds-ev.de/SN/sn2019-01.pdf (17.6. 2019).

Senta Trömel-Plötz. In: Wikipedia, die freie Enzyklopädie. Unter: www.wikiwand.com/de/Senta-Trömel-Plötz (22.11. 2019)

Stop Gendersprache jetzt! unter: https://stop-gendersprachejetzt.de (Stand 17.09.2019)

Martenstein, Harald: Schlecht, schlechter, Geschlecht. In: Die Zeit online vom 6. Juni 2013, unter: www.zeit.de/2013/24/ genderforschung-kulturelle-unterschiede (Stand 24.06. 2019). 